\title{
Two Sides of Child Maltreatment: from Psychopathic Traits to Altruistic Attitudes Inhibition
}

\author{
Filipa Carvalho $^{1} \cdot$ Laura Maciel $^{1} \cdot$ Miguel Basto-Pereira $^{2}$ (B)
}

(C) Springer Nature Switzerland AG 2019

\begin{abstract}
Child maltreatment is a known risk factor for criminal behavior, however, only a few studies have addressed the relationship between child maltreatment and the development of psychopathic traits. Meanwhile, the effect of adverse childhood experiences on prosocial behavior is practically unknown. The current research aims to explore the relationship between child maltreatment, psychopathic traits and altruistic attitudes among young adults. Six hundred and seventy-three young adults from the community filled out the Adverse Childhood Experience Questionnaire, the Youth Psychopathic Traits Inventory - Short Version and the Altruistic Attitudes Scale. Results suggest that child maltreatment is related to both psychopathic traits and inhibition of altruistic altitudes. Each adverse childhood experience appears to be associated with the development of specific forms of psychopathic traits and/or altruistic attitudes. Early identification of maltreatment is essential for prevention of antisocial behavior and for the promotion of altruistic attitudes. Research, political and social recommendations are suggested.
\end{abstract}

Keywords Child maltreatment $\cdot$ Abuse and neglect $\cdot$ Psychopathic traits $\cdot$ Prosocial behavior, altruistic attitudes

According to the World Health Organization (WHO 2018), per year, 40 million youths under 15 years old are victims of violence. In Portugal, as stated by Child Protective Services (CPS), 68,300 cases were opened in 2010, most of which concerning neglect, exposure to deviant behavior, school drop-out, and psychological abuse. The link between maltreatment and antisocial behavior is well-known in the literature. Braga et al. (2017) conducted a meta-analysis consisting of 33 prospective longitudinal studies, including 23,973 youths. This study indicated that all types of child maltreatment are independent longitudinal predictors of aggressive antisocial behavior during adolescence. Similarly, Fox et al. (2015) studied 22,575 individuals involved in the juvenile justice system and concluded that adverse childhood experiences increased the likelihood of violent crimes recidivism by $35 \%$.

According to Cicchetti and Toth (2005) Developmental Psychopathology Theory, certain life events - especially traumatic ones - might disrupt a healthy development, resulting in

Miguel Basto-Pereira

miguelbastopereira@hotmail.com; mbpereira@ispa.pt

1 ISPA - Instituto Universitário, Rua Jardim do Tabaco 34, 1149-041 Lisbon, Portugal

2 William James Center for Research, ISPA-Instituto Universitário, Lisbon, Portugal an increased risk of dysfunctional behaviors occurrence. Children who suffer from maltreatment can develop insecure attachment relationships, which has consequences in self-image and emotional regulation (Kochanska and Kim 2013). In addition, Lee and Hoaken's (2007) suggests that extended exposure to stress caused by traumatic events may lead to a dysregulated biological stress system (i.e., cortisol abnormal release, adrenaline) and condition the development of brain structures (e.g., limbic system, prefrontal cortex) responsible for emotional regulation and self-control processes, essential in social interactions and subsequent personality development. Even though the impact of maltreatment experiences on emotional regulation has been broadly addressed in the literature, fewer studies have thoroughly explored the relationship between emotional regulation and psychopathic traits, an essential predictor of antisocial behavior. Recently, Donahue et al. (2014) recent study focused on emotional regulation disruptions as one of the central mechanisms for psychopathic traits development.

Psychopathic traits are absolutely crucial to an in-depth understanding of antisocial behavior. In this regard, Matt DeLisi (2009) states that "psychopathy is the purest and the best explanation of antisocial behavior ... it mirrors the elemental nature and embodies the pejorative essence of antisocial behavior" (p. 256). Psychopathy refers to a constellation of affective (e.g., emotional callousness), interpersonal (e.g., 
grandiosity; manipulation), and behavioral (e.g., irresponsibility; impulsivity) traits, present in a wide range of antisocial behaviors (DeLisi 2009; Van Baardewijk et al. 2010). These traits highly predict criminal behavior and are related to child and juvenile development. Psychopathy assessment tools (e.g., Historical, Clinical, Risk Management-20; Psychopathy Checklist or Hare Psychopathy Checklist-Revised) are successfully used to predict criminal recidivism (DeLisi 2009).

Although there is consistent scientific evidence that abused children have, on average, higher levels of psychopathic traits (e.g. Kimonis et al. 2013), it is still largely unknown how distinctive types of child maltreatment influence different domains of psychopathic traits. It is plausible that different types of abuse, due to their specific nature, have differentiated effects on brain structures' development and maturation, and consequently contribute to psychopathic traits development in a specific way. For example, if an impoverished emotional environment is what defines emotional neglect, it is conceivable that neglected children exhibit more distinguished emotional psychopathic traits such as callousness. Likewise, emotional abuse might be more related to an interpersonal dimension of psychopathy, namely traits of manipulation. However, this particular domain remains underexplored.

\section{Child Maltreatment and Prosocial Behavior: the Hypothesis of Altruism Inhibition}

Prosocial involvement can be broadly defined as the natural tendency and voluntary act of benefiting others (Lam 2012). Given that empirical studies have shown adverse childhood experiences to increase the risk of developing an antisocial course of life, does it also inhibit prosocial behavior? If we take into account that abused children present an increased risk for low empathy (Famularo et al. 1992; Kaufman and Cicchetti 1989), namely constraints on understanding emotion (Shipman and Zeman 1999), higher levels of aggressiveness (Braga et al. 2017) and general delinquency (Basto-Pereira et al. 2016), there is likely less propensity to act prosocially. However, as far as we know, this hypothesis has not been tested.

Altruistic attitudes are one of the best representations of prosocial involvement. Altruism can be defined as attitudes that contribute "to the well-being of others at some cost to themselves and without expectation of reward" (Loureiro and Lima 2009, p. 74). To the limit of our knowledge, the only study to explore the relationship between child maltreatment and altruistic attitudes was conducted by Music (2011), with a qualitative approach and a sample of only two children. This study suggests that lower interest in others is one of the main effects of child maltreatment, meaning that children are less capable of caring for others and, consequently, are less altruistic, since the ability to care for others coexists with other skills such as feeling affection, postponing gratification and maintaining peer relationships. In cases of child maltreatment, a normative development of these skills may be affected and children might have little moral sense, empathy or affection, and demonstrate little effort to fit into social expectations.

To the best of our knowledge, this is the first empirical study to analyze the relationship between adverse childhood and adolescence experiences and altruism. Therefore, we have two main aims, to explore the specific role of physical, sexual and emotional abuse, as well as, physical and emotional neglect during childhood and adolescence on; a) psychopathic traits domains and b) altruistic attitudes during early adulthood.

\section{Method}

\section{Participants}

The current study includes 673 young adults from the community, between the age of 18 and 20 years old. Two hundred and forty-four $(36.31 \%)$ participants were male and 428 $(63.69 \%)$ were female and one missing value for gender, with a mean age of $18.90(\mathrm{SD}=0.82)$. Regarding occupational activities, the sample majority were students (77.38\%), 9.97\% work and study, $2.98 \%$ have no particular type of activity and $9.67 \%$ work and are financially autonomous. The mean number of school years completed was $11.43(\mathrm{SD}=1.26)$. A nonprobabilistic sample was collected in various locations, including high schools, professional and vocational schools, universities, workplaces and volunteer, sports and recreational organizations throughout the country. Only $4 \%(n=29)$ of the individuals invited to participate either declined or quit during completion of the evaluation protocol, indicating a participation refusal rate of less than 5\%. A detailed description of demographic characteristics is presented in Table 1.

\section{Measures}

Social and Family Situation Questionnaire This questionnaire was used to collect sociodemographic and family situation data, including gender, age, school years, ethnic group, occupation, household dimension and other general information.

Adverse Childhood Experiences Questionnaire (Felitti et al. 1998; Portuguese Version, Silva and Maia 2008) This instrument evaluates history of adverse childhood experiences, including abuse and neglect. It is a self-report questionnaire for adults, consisting on three categories: experiences of abuse, negligence and dysfunctional family environment. Abuse and neglect experiences or exposure to domestic violence were classified according to their frequency through a 5-point 
Table 1 Sociodemographic characteristics

\begin{tabular}{lll}
\hline Characteristics & $n$ & $\%$ \\
\hline Gender & & \\
Male & 244 & $36.31 \%$ \\
Female & 428 & $63.69 \%$ \\
Age (M/SD) & 18,90 & 0.82 \\
School years (M/SD) & 11,43 & 1.26 \\
Socioeconomic status & & \\
Low & 234 & $35.67 \%$ \\
Medium & 331 & $50.46 \%$ \\
High & 91 & $13.87 \%$ \\
Ethnicity & & \\
Portuguese with portuguese ancestry & 569 & $84.80 \%$ \\
Portuguese with african ancestry & 56 & $8.35 \%$ \\
Portuguese gypsy community & 0 & $0.00 \%$ \\
Portuguese with another ancestry & 30 & $4.47 \%$ \\
Immigrant community & 16 & $2.38 \%$ \\
Skin color & & \\
White & 606 & $91.27 \%$ \\
Black & 38 & $5.72 \%$ \\
Other & 20 & $3.01 \%$ \\
Occupation & & \\
Worker & 65 & $9.67 \%$ \\
Student & 520 & $2.98 \%$ \\
Student - Worker & 67 & \\
Without occupation & 20 & \\
\hline & & \\
& &
\end{tabular}

Likert scale, where response options range between " 1 . Never" and "4. Too Often". The remaining experiences are evaluated taking into account a dichotomous scale of "Yes" or "No." For this study, the five experiences of child abuse and neglect were used. Emotional abuse was identified by the occurrence of situations related to injuries or in which feelings of fear were provoked, including threats of physical violence. Physical abuse was described by events in which an adult hit a youngster violently, leaving visible marks (e.g., "How often did they hit you?"). Sexual abuse was characterized by the presence of sexual experiences between a child or teenager under the age of 18 and a person at least five years older (e.g., a family member, friend or stranger), and was calculated through dichotomous questions (e.g., "Did an adult touch or caress your body in a sexualized way?"). Physical neglect was evaluated as the absence or passive response to the basic needs of the child, both physical and biological. Evaluated through direct and inverted questions, this dimension verifies the frequency of such experiences (e.g., "My parents were too drunk or disturbed to take care of our family"). Finally, emotional neglect was considered present when there was no response to the child's emotional needs (e.g., "There was someone in my family who helped me feel special or important"). This questionnaire presents appropriate psychometric characteristics (Felitti et al. 1998; Silva and Maia 2008).

Youth Psychopathic Inventory - Short Version (YPI-S; Van Baardewijk et al. 2010; Portuguese Version: Pechorro et al. 2015) This questionnaire consists of 18 items that can be scored on a 4-point Likert scale, ranging from "1. Strongly disagree" to "4. Strongly agree". In order to evaluate psychopathic traits, the instrument presents a global dimension and is also subdivided into three dimensions, namely the Interpersonal Dimension, that comprises traits of dishonest charm, manipulation, lying and grandiosity (e.g., "I can deceive people using my charm and smile"), the Affective Dimension, that refers to traces of insensitivity, callousness and lack of remorse (e.g., "When other people have problems, it is often their fault, so we shouldn't help them"), and the Behavioral Dimension, characterized by impulsivity, irresponsibility and sensation seeking (e.g., "I consider myself a rather impulsive person"). Both the original (Van Baardewijk et al. 2010) and Portuguese (Pechorro et al. 2015) versions show adequate psychometric characteristics. Dimensions' internal consistency ranged from $\alpha=$ 0.72 to $\alpha=0.75$, and the total scale's internal consistency was $\alpha=0.79$, and therefore considered appropriate.

Altruistic Attitudes Scale (Loureiro and Lima 2009) This psychological instrument was developed in Portugal and aims to evaluate altruistic attitudes in their cognitive, affective and behavioral components. It is composed of 12 items, subdivided into three subscales, correspondent to the three components just mentioned. Therefore, the Cognitive Dimension refers to individual's beliefs and perceptions (e.g., "I think that in this world everyone should just worry about themselves"), the Affective Dimension assesses emotional responses (e.g., "Caring for someone without expecting a reward"), whereas the Behavioral Dimension relates to participants' behavioral commitments (e.g., "Helping a colleague when my knowledge is greater than his, even though I don't know him very well'). Answers are scored through a 5-point Likert scale. The original version presents appropriate psychometric characteristics. Internal consistency obtained for the global scale was $\alpha=0.74$, which is considered satisfactory, and for each dimension internal consistency varies between $\alpha=0.61$ and $\alpha=0.84$, which can be considered acceptable.

\section{Procedures}

After being advised of the research goals, existence of confidentiality and possibility of withdrawing at any time, the participants signed an informed consent and the protocol was self-administered in groups. The average response time was approximately $25 \mathrm{~min}$. Subsequent to data collection, a database was created in IBM SPSS Statistics version 25.0 
(Chicago IL, USA) program. Data analysis was then carried out using Pearson correlations (between quantitative variables) and Pearson point biserial correlations (between quantitative and dichotomous variables), in order to test associations between experiences of abuse and anti/pro-social indicators in early adulthood. Posteriorly, multiple linear regressions were conducted to analyze the predictive capacity of each form of child and adolescent maltreatment experiences in those same indicators, after adjusting other experiences of maltreatment and sociodemographic variables. This study was approved by ISPA - Instituto Universitário Ethics Committee, as part of the "International Study of Pro/Antisocial Behavior in Young Adults" (SOCIALDEVIANCE1820) - Portuguese sample.

\section{Results}

Results were organized into two phases; in the first phase, correlations between child maltreatment experiences, psychopathic traits and altruistic attitudes were analyzed. Secondly, child abuse and neglect were tested as predictors of psychopathic traits and altruistic attitudes during early adulthood, after controlling other forms of maltreatment and potentially confounding variables. It was found that 26 correlations were statistically significant out of 40 possible correlations between different forms of child maltreatment and anti/prosocial patterns. Interestingly, all forms of abuse (physical, sexual and emotional) and neglect (physical and emotional) were correlated with at least with one indicator of psychopathic traits and with one indicator of altruistic attitudes. Pearson correlations (for correlations between quantitative variables) and point biserial correlations (for correlations between quantitative and dichotomic variables) are presented in Table 2.

In order to understand which experiences of child maltreatment associate with psychopathic traits and altruistic attitudes, multivariate linear regression models were conducted following adjustment of other maltreatment experiences and sociodemographic variables. These models included sociodemographic/potentially confounding variables (age, gender, race/ethnicity, socioeconomic status) variables in Step 1 and each maltreatment experience, namely abuse (physical, sexual and emotional) and neglect (physical and emotional) in Step 2. Physical abuse, $\beta=.090, p<.050$, emotional neglect, $\beta=.092, p=.044$, and sexual abuse, $\beta=.112$, $p=.006$, were statistically significant predictors of the total dimension of psychopathic traits, after adjusting the remaining variables. The model explained $10.3 \%$ of the variance, $R^{2}=.103, F(9,596)=7.645, p<.001$.

Regarding each subscale, the Affective Dimension (callous/unemotional domain) of psychopathic traits, only emotional neglect, $\beta=.130, p=.004$, was a statistically significant predictor, and the general model explained $12.2 \%$ of the variance, $R^{2}=.122, F(9,598)=9264, p<.001$. Regarding interpersonal dimension of psychopathy (manipulation/grandiosity), only sexual abuse, $\beta=.108, p=.009$, was a statistically significant predictor, explaining only $4.7 \%$ of the variance, $R^{2}=0.047, F(9,600)=3.289, p=0.001$. Further, physical, $\beta=.118, p=.011$, and sexual abuse, $\beta=.108, p=.009$ ) were statistically significant predictors of the behavioral dimension (impulsivity/irresponsibility domain), and the final model explained $7.9 \%$ of the variance, $R^{2}=.079, F(9,600)=$ 5.694, $p<.001$ (Table 3).

Concerning the altruistic attitudes total scale, only emotional neglect, $\beta=-.18, p<.001$, was a statistically significant (and negative) predictor, and the final model explained $7.6 \%$ of the variance, $R^{2}=.076, F(9,593=5.404, p<.001$. Regarding each domain, the Affective Dimension was inversely predicted by emotional neglect, $\beta=-.203, p<.001$, in which $8.7 \%$ of the variance was explained by the model, $R^{2}=.087, F(9,598)=6.315, p<.001$; and the Behavioral Dimension was negatively predicted by physical abuse, $\beta=$ $-0.098, p=.038$, and emotional neglect $\beta=-.134, p=.005$ ), the final model explained $4.7 \%$ of the variance, $R^{2}=.047$, $F(9,598)=3.268, p=0.001$. None of the maltreatment experiences was a statistically significant predictor of the Cognitive Dimension (Table 4).

\section{Discussion}

This research aimed to explore the relationship between experiences of child and adolescent abuse and neglect in psychopathic traits and pro-social attitudes in early adulthood. As far as we know, this is the first quantitative study analyzing the relationship between different forms of child maltreatment and altruistic attitudes in young adults. Also, scarce investigation has been conducted to study the relationship between psychopathic traits and different forms of early maltreatment. The fact that this study includes young adults is particularly important, since it is at this stage of life that delinquent behavior reaches its peak (Stolzenberg and D'Alessio 2008).

First, this study intended to understand if maltreatment experiences, namely abuse and neglect, are related to the development of psychopathic traits. Sexual abuse, physical abuse and emotional neglect were found to be related to psychopathic traits in general. These results are in line with Beach et al.' (2010) and Barnett's (1997) findings that abuse and neglect are important risk factors for persistent antisocial patterns. Perhaps more important, these results show that specific adverse experiences are associated with different dimensions of psychopathic traits, suggesting that different forms of abuse and neglect have an impact on the child's healthy development, in specific ways. Whereas physical abuse was related with the behavioral dimensions of psychopathic traits, emotional abuse was related with the callous/unemotional traits, the affective domain of psychopathy. Thus, this study suggests 
Table 2 Correlation matrix between variables

\begin{tabular}{|c|c|c|c|c|c|c|c|c|}
\hline \multirow[t]{2}{*}{ Variables } & \multicolumn{4}{|c|}{ Psychopathic Traits } & \multicolumn{4}{|c|}{ Altruistic Attitudes } \\
\hline & Total & GM & II & $\mathrm{CU}$ & Total & Cognitive & Affective & Behavioral \\
\hline Gender & $-.19^{* *}$ & $-.16^{* *}$ & .01 & $-.29^{* * *}$ & $.11^{* * *}$ & .03 & $.18^{* *}$ & .05 \\
\hline Age & -.05 & .01 & -.05 & -.07 & .07 & .04 & .01 & $.11^{* *}$ \\
\hline SES & -.01 & -.05 & .03 & .02 & $-.11^{* *}$ & $-.12^{* *}$ & -.00 & $-.09^{*}$ \\
\hline Ethnicity & $.12^{* *}$ & $.08^{*}$ & $.09^{*}$ & .08 & -.02 & .02 & -.07 & -.01 \\
\hline Physical Abuse & $.17^{* *}$ & $.09^{*}$ & $.18^{* *}$ & $.11^{* * *}$ & -.04 & -.05 & $-.08^{*}$ & .05 \\
\hline Sexual Abuse & $.16^{* *}$ & $.12^{* *}$ & $.17^{* *}$ & .06 & -.04 & -.03 & $-.08^{*}$ & .01 \\
\hline Emotional Abuse & $.12^{* *}$ & .03 & $.14^{* * *}$ & $.09^{*}$ & -.07 & $-.08^{*}$ & -.04 & -.04 \\
\hline Emotional Neglect & $.16^{* *}$ & .05 & $.13^{* *}$ & $.18^{* * *}$ & $-.21^{* *}$ & $-.12^{* *}$ & $-.20^{* *}$ & $-.12^{* *}$ \\
\hline Physical Neglect & $.11^{* * *}$ & .03 & $.09^{*}$ & $.13^{* *}$ & $-.12^{* *}$ & $-.12^{* *}$ & $-.13^{* *}$ & -.01 \\
\hline Total Psychopathic Traits & & $.76^{* * *}$ & $.72^{* * *}$ & $.68^{* * *}$ & $-.28^{* *}$ & $-.28^{* *}$ & $-.22^{* *}$ & $-.09^{*}$ \\
\hline GM Psychopathic Traits & & & $.29^{* * *}$ & $.25^{* *}$ & $-.10^{*}$ & $-.10^{*}$ & $-.14^{* *}$ & .02 \\
\hline II Psychopathic Traits & & & & $.29^{* *}$ & $-.14^{* *}$ & $-.20^{* *}$ & -.06 & -.05 \\
\hline FE Psychopathic Traits & & & & & $-.38^{* *}$ & $-.33^{* *}$ & $-.30^{* *}$ & $-.19^{* *}$ \\
\hline Total Altruistic Attitudes & & & & & & $.67^{* *}$ & $.71^{* *}$ & $.75^{* *}$ \\
\hline Cognitive Altruistic Attitudes & & & & & & & $.19^{* *}$ & $.19^{* *}$ \\
\hline Affective Altruistic Attitudes & & & & & & & & $.39^{* * *}$ \\
\hline Behavioral Altruistic Attitudes & & & & & & & & - \\
\hline
\end{tabular}

Gender $($ male $=0$; female $=1)$; SES = Socioeconomic status $($ medium $/$ high $=0 ;$ low $=1)$; Ethnicity $($ majority = $0 ;$ minority $=1) ; \mathrm{GM}=\mathrm{Grandiose}-$ Manipulative dimension; II = Impulsive-Irresponsible dimension; CU = Callous-Unemotional dimension; * $p<.05$. ** $p<.001$

Table 3 Multiple linear regression to predict domains of psychopathic traits

\begin{tabular}{|c|c|c|c|c|c|c|c|c|c|c|c|c|c|}
\hline \multirow[t]{3}{*}{ Model } & \multirow[t]{3}{*}{ Variables } & \multicolumn{12}{|c|}{ Linear Regression } \\
\hline & & \multicolumn{3}{|c|}{ Psychopathic Traits - Total } & \multicolumn{3}{|c|}{ Psychopathic Traits - GM } & \multicolumn{3}{|c|}{ Psychopathic Traits - II } & \multicolumn{3}{|c|}{ Psychopathic Traits - CU } \\
\hline & & $\mathrm{b}$ & SE & $\beta$ & $\mathrm{b}$ & SE & $\beta$ & $\mathrm{b}$ & SE & $\beta$ & $\mathrm{b}$ & SE & $\beta$ \\
\hline \multirow[t]{5}{*}{1} & Gender & -.38 & .08 & $-.18^{* *}$ & -.30 & .08 & $-.15^{* *}$ & .04 & .08 & .02 & -.61 & .08 & $-.29^{* *}$ \\
\hline & Age & -.06 & .05 & -.05 & .01 & .05 & .01 & -.06 & .05 & -.05 & -.09 & .05 & $-.08^{*}$ \\
\hline & SES & .01 & .08 & .01 & -.09 & .08 & -.05 & .09 & .08 & .04 & .05 & .08 & .02 \\
\hline & Ethnicity & .37 & .11 & $.14 * *$ & .19 & .11 & .07 & .31 & .11 & $.11^{*}$ & .27 & .11 & $.10^{*}$ \\
\hline & $\mathrm{R}^{2}$ & .05 & & & .03 & & & .02 & & & .10 & & \\
\hline \multirow[t]{10}{*}{2} & Gender & -.35 & .08 & $-.17^{* *}$ & -.30 & .08 & $-.14^{* *}$ & .08 & .08 & .04 & -.57 & .08 & $-.27^{* *}$ \\
\hline & Age & -.06 & .05 & -.05 & .01 & .05 & .01 & -.06 & .05 & -.05 & -.10 & .05 & $-.08^{*}$ \\
\hline & SES & -.04 & .08 & -.02 & -.11 & .08 & -.05 & .04 & .08 & .02 & -.00 & .08 & -.00 \\
\hline & Ethnicity & .32 & .11 & $.12^{* *}$ & .15 & .11 & .06 & .25 & .11 & $.09^{*}$ & .26 & .11 & $.09^{*}$ \\
\hline & Physical Abuse & .26 & .13 & $.09^{*}$ & .16 & .14 & .06 & .34 & .13 & $.12^{*}$ & .13 & .13 & .04 \\
\hline & Sexual Abuse & .34 & .12 & $.11^{*}$ & .33 & .13 & $.11^{*}$ & .32 & .12 & $.11^{*}$ & .05 & .12 & .02 \\
\hline & Emotional Abuse & .05 & .12 & .02 & -.05 & .12 & -.02 & .18 & .12 & .07 & -.03 & .12 & -.01 \\
\hline & Emotional Neglect & .32 & .16 & $.09^{*}$ & .05 & .16 & .02 & .19 & .16 & .06 & .45 & .16 & $.13^{*}$ \\
\hline & Physical Neglect & .06 & .13 & .02 & -.00 & .14 & -.00 & .09 & .13 & .03 & .10 & .13 & .03 \\
\hline & $\mathrm{R}^{2}$ & .10 & & & .05 & & & .08 & & & .12 & & \\
\hline
\end{tabular}

$\mathrm{Z}$ score standardization was applied to the dependent variables; Gender $($ male $=0 ;$ female $=1) ; \mathrm{SES}=$ Socioeconomic status $(\mathrm{médium} / \mathrm{high}=0 ;$ low $=1)$; Ethnicity (majority = 0; minority = 1); GM = Grandiose-Manipulative dimension; II = Impulsive-Irresponsible dimension; CU = Callous-Unemotional dimension; * $p<.05$. ** $p<.001$ 
Table 4 Multiple linear regression to predict altruistic attitudes

\begin{tabular}{|c|c|c|c|c|c|c|c|c|c|c|c|c|c|}
\hline \multirow[t]{3}{*}{ Model } & \multirow[t]{3}{*}{ Variables } & \multicolumn{12}{|c|}{ Linear Regression } \\
\hline & & \multicolumn{3}{|c|}{ AA - Total } & \multicolumn{3}{|c|}{ AA - Cognitive } & \multicolumn{3}{|c|}{ AA - Affective } & \multicolumn{3}{|c|}{ AA - Behavioral } \\
\hline & & $\mathrm{b}$ & SE & $\beta$ & $\mathrm{b}$ & SE & $\beta$ & $\mathrm{b}$ & SE & $\beta$ & $\mathrm{b}$ & $\mathrm{SE}$ & $\beta$ \\
\hline \multirow[t]{5}{*}{1} & Gender & .31 & .09 & $.15^{* *}$ & .14 & .09 & .07 & .40 & .09 & $.19^{* *}$ & .16 & .08 & .07 \\
\hline & Age & .10 & .05 & $.08^{*}$ & .03 & .05 & .03 & .03 & .05 & .03 & .16 & .05 & $.13^{* *}$ \\
\hline & SES & -.26 & .09 & $-.12^{\text {*** }}$ & -.30 & .08 & $-.14^{* *}$ & -.02 & .09 & -.01 & -.19 & .09 & -.09 \\
\hline & Ethnicity & -.06 & .11 & -.02 & .06 & .11 & .02 & -.22 & .11 & $-.08^{*}$ & -.02 & .11 & -.01 \\
\hline & $\mathrm{R}^{2}$ & .04 & & & .02 & & & .04 & & & .03 & & \\
\hline \multirow[t]{10}{*}{2} & Gender & .26 & .09 & $.12^{* *}$ & .10 & .09 & .05 & .35 & .09 & $.17^{* * *}$ & .14 & .09 & .07 \\
\hline & Age & .11 & .05 & $.09^{*}$ & .03 & .05 & .03 & .04 & .05 & .03 & .15 & .05 & .13 \\
\hline & SES & -.18 & .09 & $-.09^{*}$ & -.25 & .09 & $-.12^{* *}$ & .06 & .09 & .03 & -.16 & .09 & -.07 \\
\hline & Ethnicity & -.07 & .11 & -.03 & .05 & .11 & .02 & -.22 & .11 & $-.08^{*}$ & -.06 & .11 & -.02 \\
\hline & Physical Abuse & .15 & .14 & .05 & .05 & .14 & .02 & .01 & .14 & .00 & .29 & .14 & $.10^{*}$ \\
\hline & Sexual Abuse & -.05 & .13 & -.02 & -.04 & .13 & -.01 & -.13 & .13 & -.04 & .05 & .13 & .02 \\
\hline & Emotional Abuse & -.02 & .12 & -.01 & -.13 & .12 & -.05 & .10 & .12 & .04 & -.03 & .12 & -.01 \\
\hline & Emotional Neglect & -.63 & .16 & $-.18^{* *}$ & -.18 & .16 & -.05 & -.71 & .16 & $-.20^{* * *}$ & -.46 & .16 & $-.13^{*}$ \\
\hline & Physical Neglect & -.13 & .14 & -.04 & -.22 & .14 & -.07 & -.09 & .14 & -.03 & .05 & .14 & .02 \\
\hline & $\mathrm{R}^{2}$ & .08 & & & .04 & & & .07 & & & .05 & & \\
\hline
\end{tabular}

$\mathrm{Z}$ score standardization was applied to the dependent variables; $\mathrm{AA}=$ Altruistic Attitudes; Gender (male $=0$; female $=1$ ); $\mathrm{SES}=$ Socioeconomic status $($ medium $/$ high $=0$; low $=1)$; Ethnicity (majority $=0$; minority $=1) ; * p<.05 . * * p<.001$

that each adverse experience plays different roles on the development of persistent antisocial patterns as proposed by Basto-Pereira et al. (2016). These results may explain why the diversity of adverse childhood experienced is one of the best predictors of juvenile delinquency and adult crime (e.g., Fox et al. 2015). For instance, continued physical abuse may affect brain structures related to self-control and lead to a hypervigilance to potential threats and non-rational responses to normal stimuli that can be interpreted as danger (Lee and Hoaken 2007), influencing impulsivity levels, a behavioral trait of psychopathy. A child living in very poor emotional environment, as a victim of emotional neglect, might become callous and unemotional persons (related to the affective domain of psychopathy).

Sexual abuse was the type of maltreatment most associated with different dimensions of youth psychopathy. Sexual abuse was related to Interpersonal and Affective domains of psychopathic traits, which include traits of manipulation, dishonest charm, callousness and unemotionality. Interestingly, Braga et al.'s (2017) meta-analysis found that the main predictor of aggressive antisocial behavior was child sexual abuse. Psychopathy has a well-known relationship with extremely violent behavior (for a review, see Anderson and Kiehl 2014). This association can be both explained by the development of post-traumatic symptoms (such as anger and lack of emotion regarding others) after a sexual abuse experience, as well as the development of maladaptive beliefs and schemas for sexual behavior, which will condition both individual's cognitive and emotional development (Finkelhor and Browne 1985).

Our second aim was to explore if experiences of child and adolescent maltreatment are inhibitory factors of altruistic attitudes, in their various dimensions (i.e., cognitive, affective and behavioral). To our knowledge, this is the first quantitative study to investigate the relationship between child maltreatment and prosocial attitudes, specifically altruistic attitudes. Abuse and neglect presented a negative correlation with altruistic attitudes. Our findings support the view that different forms of maltreatment are related, in opposite ways, to psychopathic traits and altruistic attitudes. As expected, this study suggests that a child or adolescent who has been victim of physical abuse or emotional neglect is not as likely to acquire prosocial skills when compared to a youth who experienced support, affection and has not been victim of violence. In this regard, Music (2011) suggests that lack of interest in others is an effect of youth maltreatment, revealing that adverse childhood experiences translate into less altruistic attitudes. In this context, attention, affection and care present an imperative place in establishing social bonds, providing a greater openness to prosocial behaviors.

Emotional neglect seems to be particularly important in inhibiting a normative altruism development, conditioning both affective and behavioral dimensions of altruistic attitudes. One possible explanation is that deprivation of 
affection can diminish behaviors that benefit others, since neglected children and teenagers are subjected to emotionally sterile learning environments, which may be responsible for abnormal affective functioning. Another possibility is that youths who have been victims of maltreatment, such as emotional neglect, possess greater cognitive deficits and more limitations in their peer interaction (Kaufman and Cicchetti 1989; Hoffman-Plotkin and Twentyman 1984), which would, logically, limit prosocial attitudes. However, this is a largely neglected area of scientific knowledge and only scientific studies can expand our understanding of mechanisms underlying the relationship between child and adolescent maltreatment and altruistic attitudes inhibition.

This work suggests that young adults who experienced childhood or adolescence maltreatment are less likely to possess altruistic attitudes and are more likely to exhibit psychopathic traits. In light of these results, we propose several recommendations. First, future research should focus both on prevention and intervention with families at risk. Regarding primary prevention, school and pre-school services, along with health centers, should raise awareness and prevent youth maltreatment. Concerning intervention with at-risk families, juvenile justice system, and the Child Protective Services should provide services related to mental health and parental education. We also suggest a strong cooperation between the juvenile justice system and children and youth protection systems to create delinquency prevention programs, since many youngsters shift from protection systems to the juvenile justice system and then to the adult justice system.

It should also be taken into account that the present investigation is not free of limitations. Although the considerable number of participants $(N=673)$ included a limited age range (between 18 and 20 years old), this can be an advantage, regarding the analysis of the relationship between maltreatment and anti/pro-social attitudes in early adulthood. However, this is a non-probabilistic sample, thus generalizations of these findings must be made cautiously (and even more for other age groups). Another limitation is related with the cross-sectional design of this research. Each participant, answering the protocol only at a given moment and referring to past experiences, was exposed to several contextual and mnesic factors (i.e., mnesic access to information and emotional state) that might have influenced how he/she evaluated his/her own experiences. For this reason, future studies should be conducted using a longitudinal design, in order to deepen the understanding of the consequences of child maltreatment in prosocial attitudes, from a longitudinal point of view. This research field, dedicated to consequences of child and youth maltreatment and inhibition of prosocial behaviors, such as altruism, is perhaps one of the most neglected areas of knowledge. Future studies should continue to develop this line of research.

\section{Compliance with Ethical Standards}

Conflict of Interest On behalf of all authors, the corresponding author states that there is no conflict of interest.

Ethical Standards and Informed Consent All procedures followed were in accordance with the ethical standards of the responsible committee on human experimentation [institutional and national] and with the Helsinki Declaration of 1975, as revised in 2000. Informed consent was obtained from all patients for being included in the study."

\section{References}

Anderson, N. E., \& Kiehl, K. A. (2014). Psychopathy: Developmental perspectives and their implications for treatment. Neurology and Clinical Neuroscience, 32, 103-117.

Barnett, D. (1997). The effects of early intervention on maltreating parents and theirs children. In M. J. Guralnick (Ed.), The effectiveness of early intervention (pp. 147-170). Baltimore: Paul Brookes.

Basto-Pereira, M., Miranda, A., Ribeiro, S., \& Maia, A. (2016). Growing up with adversity: From juvenile justice involvement to criminal persistence and psychosocial problems in young adulthood. Child Abuse \& Neglect, 62, 63-75.

Beach, S. R., Brody, G. H., Gunter, T. D., Packer, H., Wernett, P., \& Philibert, R. A. (2010). Child maltreatment moderates the association of MAOA with symptoms of depression and antisocial personality disorder. Journal of Family Psychology, 24(1), 12-20.

Braga, T., Gonçalves, L., Basto-Pereira, M., \& Maia, Â. (2017). Unraveling the link between maltreatment and juvenile antisocial behavior: A meta-analysis of prospective longitudinal studies. Aggression and Violent Behavior, 33, 37-50.

Cicchetti, D., \& Toth, S. (2005). Child maltreatment. Annual Review of Clinical Psychology, 1(1), 409-438.

DeLisi, M. (2009). Psychopathy is the unified theory of crime. Youth Violence and Juvenile Justice, 7(3), 256-273.

Donahue, J. J., McClure, K. S., \& Moon, S. M. (2014). The relationship between emotion regulation difficulties and psychopathic personality characteristics. Personality Disorders, 5(2), 186-194.

Famularo, R., Kinscherff, R., \& Fenton, T. (1992). Psychiatric diagnoses of maltreated children: Preliminary findings. Journal of the American Academy of Child \& Adolescent Psychiatry, 31(5), 863-867.

Felitti, V., Anda, R., Nordenberg, D., Williamson, D., Spitz, A., ... \& Marks, J. (1998). Relationship of childhood abuse and household dysfunction to many of the leading causes of death in adults. American Journal of Preventive Medicine, 14, 245-258.

Finkelhor, D., \& Browne, A. (1985). The traumatic impact of child sexual abuse: A conceptualization. American Journal of Orthopsychiatry, 55(4), 530-541.

Fox, B., Perez, N., Cass, E., Baglivio, M., \& Epps, N. (2015). Trauma changes everything: Examining the relationship between adverse childhood experiences and serious, violent and chronic juvenile offenders. Child Abuse \& Neglect, 46, 163-173.

Hoffman-Plotkin, D., \& Twentyman, C. T. (1984). A multimodal assessment of behavioral and cognitive deficits in abused and neglected preschoolers. Child Development, 55(3), 794-802.

Kaufman, J., \& Cicchetti, D. (1989). Effects of maltreatment on schoolage Children's socioemotional development: Assessments in a daycamp setting. Developmental Psychology, 25(4), 516-524.

Kimonis, E. R., Kostas, A. F., Isoma, Z., \& Donoghue, K. (2013). Maltreatment profiles among incarcerated boys with callousunemotional traits. Child Maltreatment, 18(2), 108-121. 
Kochanska, G., \& Kim, S. (2013). Early attachment organization with both parents and future behavior problems: From infancy to middle childhood. Child Development, 84(1), 283-296.

Lam, C. M. (2012). Prosocial involvement as a positive youth development construct: A conceptual review. Scientific World Journal, 4, 1-8.

Lee, V., \& Hoaken, P. (2007). Cognition, emotion, and neurobiological development: Mediating the relation between maltreatment and aggression. Child Maltreatment, 12(3), 281-298.

Loureiro, A., \& Lima, M. L. (2009). Escala de atitudes altruístas: Estudo de validação e fiabilidade [Altruistic Attitudes Scales: Validity and Reliability Study]. Laboratório de Psicologia, 7, 73-83.

Music, G. (2011). Trauma, helpfulness and selfishness: The effect of abuse and neglect on altruistic, moral and pro-social capacities. Journal of Child Psychotherapy, 37(2), 113-128.

Pechorro, P., Andershed, H., Ray, J. V., Maroco, J., \& Gonçalves, R. A. (2015). Validation of the youth psychopathic traits inventory and youth psychopathic traits inventory - Short version among incarcerated juvenile delinquents. Journal of Psychopathology and Behavioral Assessment, 37, 576-586.

Shipman, K. L., \& Zeman, J. (1999). Emotional understanding: A comparison of physically maltreating and nonmaltreating mother-child dyads. Journal of Clinical Child Psychology, 28(3), 407-417.
Silva, S., \& Maia, A. (2008). Versão Portuguesa do Family ACEQuestionnaire (Questionário de História de Adversidade na Infância). In A. P. Noronha, C. Machado, L. Almeida, M. Gonçalves, S. Martins, \& V. Ramalho (Eds.), Actas da XIII conferência avaliação psicológica: Formas e contextos. Braga: Psiquilibrios Edições.

Stolzenberg, L., \& D'Alessio, S. J. (2008). Co-offending and the agecrime curve. Journal of Research in Crime and Delinquency, 45(1), 65-86.

Van Baardewijk, Y., Andershed, H., Stegge, H., Nilsson, K. W., Scholte, E., \& Vermeiren, R. (2010). Development and tests fo short versions of the youth psychopathic traits inventory and the youth psychopathic traits inventory - Child version. European Journal of Psychological Assessment, 26, 122-128.

World Health Organization (WHO) (2018). Trauma among children who are victims of violence. Retrieved from World Health Organization website: https://www.afro.who.int/news/trauma-among-childrenwho-are-victims-violenceen/

Publisher's Note Springer Nature remains neutral with regard to jurisdictional claims in published maps and institutional affiliations. 